\title{
EVALUACIÓN ESTRUCTURAL DE LAS PIEZAS DE MADERA EN CONSTRUCCIÓN HISTÓRICA CON EL USO DE ULTRASONIDO
}

\author{
Jéssica Stéfane Alves Cunha ${ }^{1 *}$, Marcelo Nogueira1, Wedson Batista dos Santos ${ }^{1}$, José Edson de Lima Torres ${ }^{1}$ \\ 1 Universidade Federal Rural de Pernambuco (UFRPE). Rua Dom Manoel de Medeiros, S/N - Dois Irmãos, CEP: 52171-900, Recife - \\ PE, Brasil.
}

*Autor correspondiente: Jéssica Stéfane Alves Cunha, cunhajsa@gmail.com

\begin{abstract}
RESUMEN: El trabajo tuvo como objetivo evaluar la sensibilidad del método del ultrasonido en la determinación del grado de sanidad de las piezas estructurales en madera que compone la fachada de la casa grande de la Hacienda Cachoeira do Taepe, Surubim, Pernambuco, Brasil. Se evaluaron siete pilares y cada pieza fueron marcados puntos a partir de la base cada 30 centímetros en el sentido longitudinal y midió el tiempo de propagación de la onda en cada punto marcado. El pilar uno, presentó lecturas con valores inferiores a $1000 \mathrm{~m} / \mathrm{s}$, mereciendo atención en la parte inferior y central de la pieza, necesitando reparaciones puntuales. En el pilar cinco se verificaron valores superiores a $2000 \mathrm{~m} / \mathrm{s}$ casi en toda la extensión de la pieza. Los resultados apuntaron a la sensibilidad del método del ultrasonido en la evaluación del grado de degradación y comprometimiento de las piezas de madera utilizadas en estructuras y la consiguiente toma de decisión en cuanto a la restauración de la edificación, tomada por el Instituto del Patrimonio Histórico y Artístico Nacional (IPHAN).
\end{abstract}

PALABRAS CLAVES: Edificación; estructura en madera; onda ultrasónica; patrimonio histórico y cultural.

\section{STRUCTURAL EVALUATION OF THE PIECES OF WOOD IN HISTORICAL CONSTRUCTION WITH THE USE OF ULTRASOUND}

\begin{abstract}
The aim of the research was to evaluate the sensitivity of the ultrasound method in determining the degree of health of the structural pieces in wood that make up the façade of the large house of the Hacienda Cachoeira do Taepe, Surubim, Pernambuco, Brazil. Seven pillars were evaluated and each piece was marked points from the base every 30 centimeters in the longitudinal direction and measured the propagation time of the wave at each marked point. Pillar one, presented readings with values lower than $1000 \mathrm{~m} / \mathrm{s}$, deserving attention in the lower and central part of the piece, needing punctual repairs. In pillar five, values higher than $2000 \mathrm{~m} / \mathrm{s}$ were verified almost throughout the length of the piece. The results pointed to the sensitivity of the ultrasound method in the evaluation of the degree of degradation and compromise of the pieces of wood used in structures and the consequent decision making regarding the restoration of the building, taken by the Institute of Historical Heritage and National Artistic (IPHAN).
\end{abstract}

KEY WORDS: Building; wood structure; ultrasonic wave; historical and cultural heritage.

\section{INTRODUCCIÓN}

Considerando el patrimonio cultural protegido es una construcción, basada en la selección de bienes que serán gestionados por las esferas estatales. Esta "selectividad", alzada a la categoría de principio interpretativo, parte de la premisa de que no todo lo que es producido 0 apreciado por el hombre puede o debe ser preservado. El principio de la selectividad impone la discriminación entre los bienes que se preservan y se transmiten por medio de criterios establecidos en la legislación, desde el punto de vista de la representatividad y no de la exhaustividad (Dantas, 2015).
En este sentido, este patrimonio debe ser dado como herencia de toda la humanidad y así en el municipio de Surubim en Pernambuco una edificación poco conocida hasta el final de los años 70 , cuando destacado por constituir un raro ejemplar de arquitectura, siendo tumbada a nivel federal en 1980. La hacienda instalada desde el tiempo del Brasil colonia, integra un pequeño grupo de edificios remanentes de significativa arquitectura rural implantada en el Nordeste brasileño, tanto desde el punto de vista estético como histórico. Este edificio, caído en ámbito federal, está sometido en la actualidad a la incidencia de un complejo conjunto de daños interrelacionados que confiere 
el carácter de urgencia en actuar en la dirección de la estabilización del inmueble (Muraro, 2013).

La utilización de madera en las edificaciones históricasbrasileñasesnotoria, sinembargo, ladurabilidad de la madera usualmente encuentra problemas, ya que ninguna especie de madera, ni siquiera las de auténtica durabilidad natural, tiene capacidad de resistir, indefinidamente, a las variaciones de las condiciones ambientales, al ataque de microorganismos, insectos xilófagos y al exceso de cargas (Abreu et al., 2013).

Los problemas interrelacionados a la durabilidad y solidez de la madera que se encuentra con asiduidad en estructuras de edificios históricos son de difícil visualización en virtud de décadas de exposición a agentes xilófagos sin las debidas prácticas de mantenimiento y conservación de las edificaciones. Estos agentes químicos y físicos actúan conjuntamente a los biológicos en la madera, acelerando el proceso de deterioración. En este sentido, es de suma importancia la aplicación de un método que permita evaluar las condiciones internas de las piezas sin aniquilarlas, es decir, la elaboración de un diagnóstico relacionado al estado de conservación de las piezas que componen la estructura, no exclusivamente para que la edificación puede ser aprovechada, pero como un bien cultural, debe ser conservada y preservada para que la posibilidad de construir un pasado, todavía se encuentra en el presente y sean llevadas al futuro.

El método encuentra gran potencial de uso también en piezas estructurales de edificaciones históricas. La velocidad de propagación de ondas en sólidos con defectos disminuye porque la onda evita el defecto, haciendo que el tiempo de propagación aumente. Las ondas son afectadas por la presencia de materiales con diferentes características de impedancia acústica de forma que la presencia de material deteriorado o diferenciado (detección de la inclinación de las fibras, de ataques biológicos, de la presencia de defectos como empenamientos, nudos, etc.) dentro de las piezas de madera acarrearán variación en la velocidad de propagación de la onda y en la amplitud de la señal emitida (Secco et al., 2012).

Las piezas de madera que por ventura posean un nivel de ataque más drástico de agentes xilófagos 0 desgaste natural necesitan la sustitución parcial 0 total de esas piezas, muchas veces componiendo una edificación de elevada representatividad histórica de la localidad, no obligatoriamente comprometiendo la composición original de las estructuras. Según Oliveira (2009), se pueden sustituir los componentes antiguos por otros tecnológicamente más apropiados, manteniendo las características arquitectónicas originales.

En esa situación, el empleo de un método que realice un diagnóstico conectado al estado de conservación del material que componen la estructura, no únicamente para que la edificación pueda ser disfrutada, sino como un bien cultural visando una perpetuación de la edificación es fundamental. En estos métodos no destructivos, el ultrasonido se fundamenta en la observación de propagación de una onda y la conexión con las constantes elásticas, esa onda acústica tiene una frecuencia superior a $20.000 \mathrm{~Hz}$ y su velocidad depende de las constantes elásticas y de la toma de tiempo de propagación de la onda; y por consiguiente, de su velocidad, permite indirectamente estimar esas constantes elásticas (Ballarin y Nogueira, 2005; Nogueira y Ballarin, 2008; Stangerlim et al., 2010; Secco et al., 2012).

Este método presenta la ventaja de no extraer cuerpo de prueba para caracterizar la madera, ya que la evaluación se realiza en la propia pieza o estructura (Martins, 2011). Esta técnica puede ser considerada eficaz para establecer un diagnóstico sobre el estado de deterioro de una estructura de madera permitiendo estimar su grado de deterioro (Liñán et al., 2011; Gatto et al., 2012; Weiler et al., 2013).

Así, el presente trabajo tuvo como objetivo diagnosticar la sanidad de piezas de material leños que componen la estructura de la fachada de edificaciones tomadas por el Patrimonio Histórico con uso de método no destructivo utilizando el ultrasonido.

\section{MATERIAL Y MÉTODOS}

La investigación fue conducida en la casa grande de la hacienda Cachoeira do Taépe (Figura 1) ubicada en el municipio de Surubim - PE, distando aproximadamente $10 \mathrm{~km}$ de la sede del municipio y 120 km de Recife - Pernambuco, Brasil (Figura 2). La casa grande fue erguida por sistema constructivo mixto, a saber, por media base de piedra que niveló el edificio (haciéndolo un sobrado), y sobre la cual se implantó el pavimento de la residencia propiamente, en estructura autónoma de madera cuyas vallas se realizaron en taipa de mano (también conocido como palo a pique - nombre extendido de la rejilla de madera que estructura el panel - taipa de sebe, de sopapo y otros) (Muraro, 2013). 
Figura 1. Vista frontal y lateral de la fachada de la Casa Grande de la Hacienda Taépe, Surubim, Pernambuco, Brasil
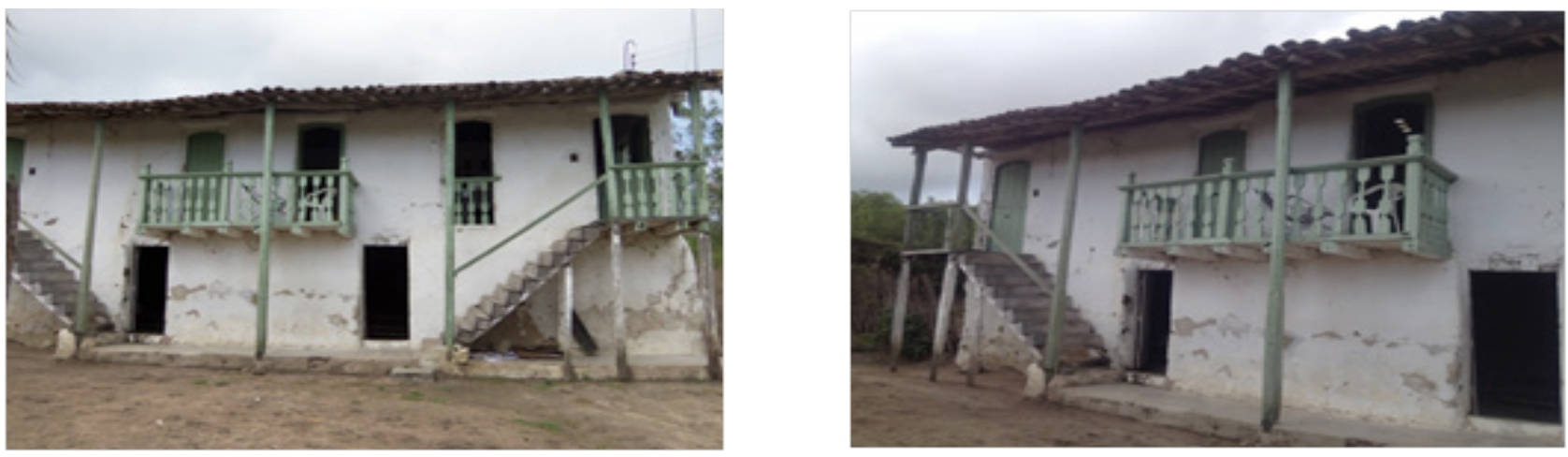

Figura 2. Ubicación de la Hacienda Cachoeira do Taépe, Surubim, Pernambuco, Brasil

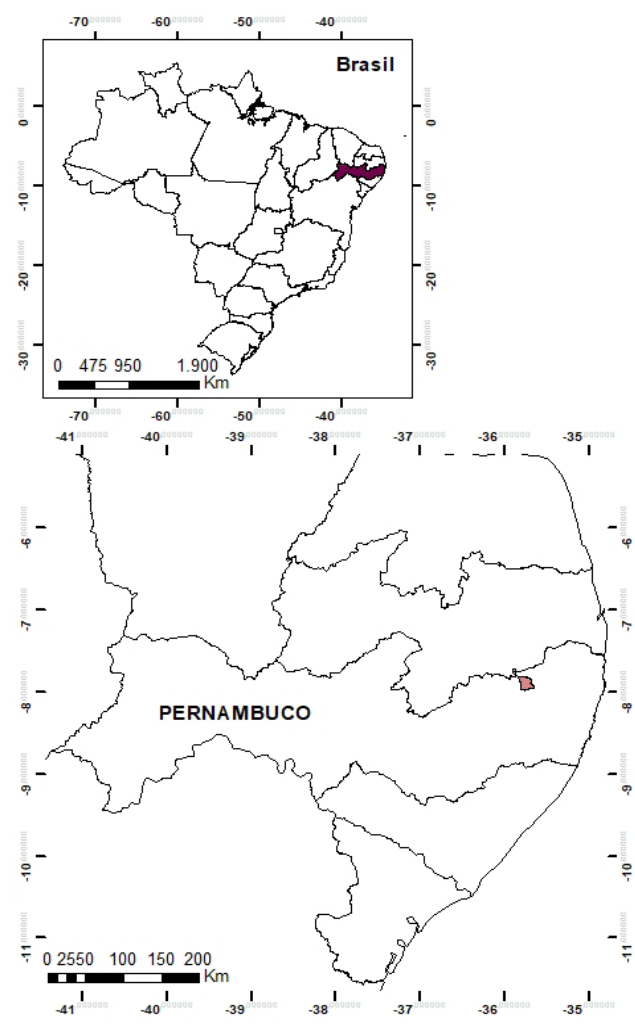

En el presente estudio, las piezas evaluadas pertenecían exclusivamente a los pilares que conformaban la fachada, teniendo como característica principal el contacto directo de las piezas de madera con el suelo, una práctica común de no preocuparse por el aislamiento de las piezas de madera en la técnica constructiva tradicional de taipa de mano del siglo XVIII.

El clima es del tipoBs'h de la clasificación Köppen, árido o semiárido, muy cálido, con precipitaciones en otoño y invierno. La temporada de lluvias normal comienza en febrero/marzo y mayo extender hasta agosto (CPRM, 2005). La temperatura, considerada alta, sigue la temporada de precipitación y el período de mayo a agosto se caracteriza por noches frías, con valores alrededor de $20^{\circ} \mathrm{C}$, asegurando un promedio

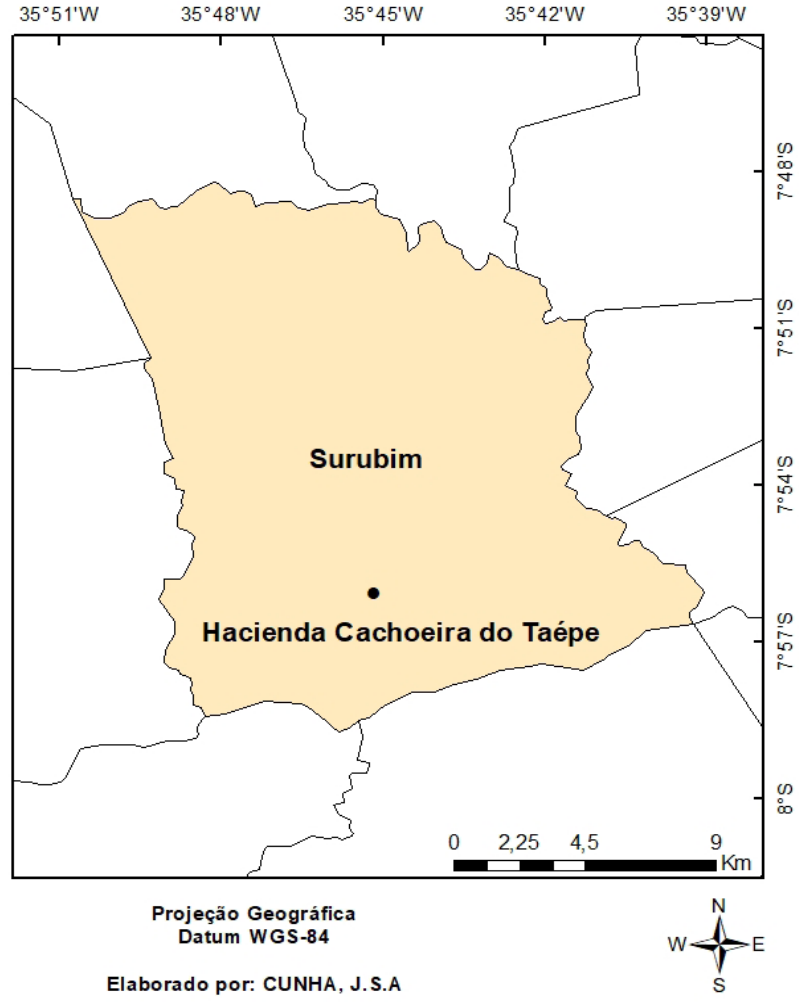

anual de $23.7^{\circ} \mathrm{C}$ y no muestra variaciones significativas durante todo el año (CPRM, 2005; Muraro, 2013).

Para el análisis, se evaluaron los siete pilares de la fachada de la casa grande de la hacienda (La nomenclatura de los pilares siguió la identificación utilizada por IPHAN - Instituto de Patrimonio Nacional Histórico y Artístico, con el pilar 1 a la derecha) y las lecturas con el medidor de humedad ha resultado en un valor medio del 10,8\%. Para cada pieza se marcaron puntos a partir de la base a cada 30 centímetros en la dirección longitudinal para tener una caracterización confiable de la pieza, donde se midió la anchura y el espesor utilizando el paquímetro digital y el tiempo de propagación de la onda ultrasónica en cada punto marcado (Figura 3). 
Figura 3. Ensayo no destructivo en la carpintería de madera fachada de la Casa Grande de la Hacienda Taépe. (a) marcación de intervalos de medición; b) tomar el tiempo de propagación de la onda; c) leer el tiempo de propagación y d) medir las dimensiones de las piezas.
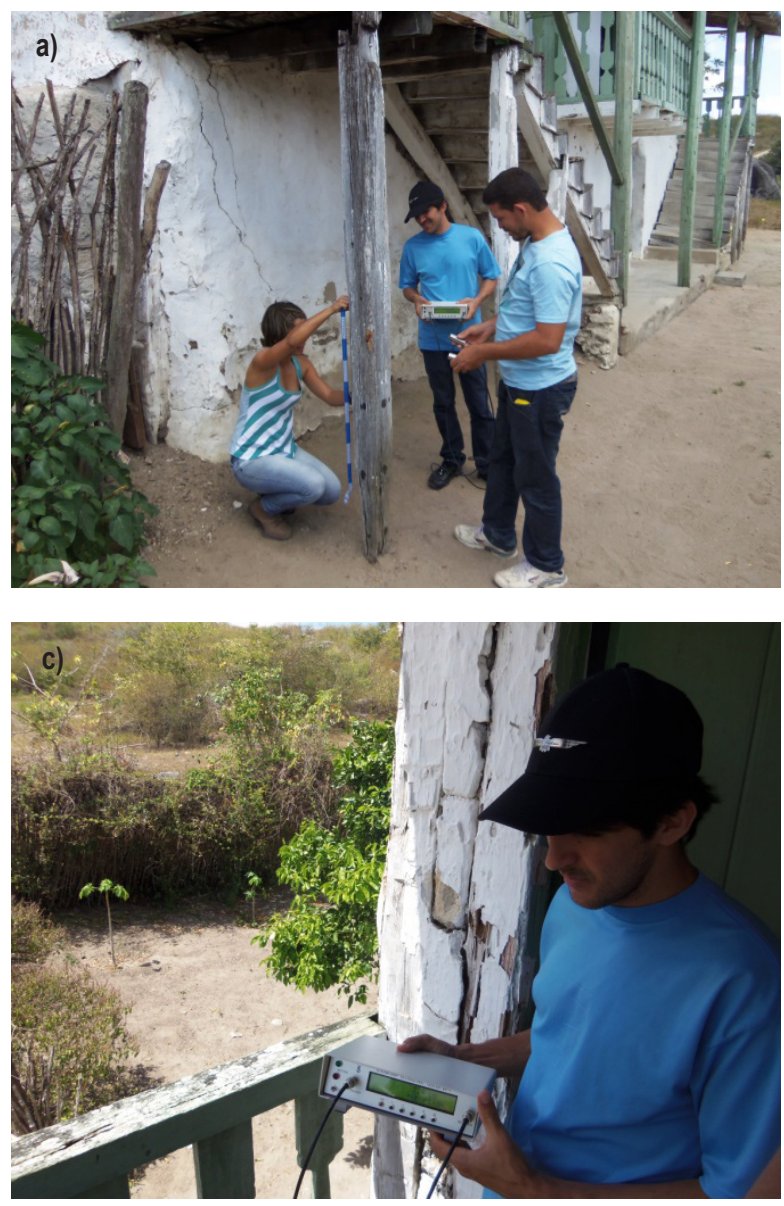

En la obtención del tiempo de propagación de la onda ultrasónica, se utilizó equipo marca Steinkamp, modelo BP-7, de fabricación alemana, y transductores piezoeléctricos planos de frecuencia $45 \mathrm{kHz}$. Se emplearon dos transductores equivalentes: uno para emisión y otro para recepción de las ondas. El equipo de ultrasonido se calibró al principio de cada serie de ensayos utilizando un cuerpo de prueba acrílico. Conforme a los procedimientos especificados en el manual de funcionamiento del equipo. Después de la calibración, justo antes de la realización del ensayo, se aplicó una fina capa de gel medicinal a las caras de los transductores, estos fueron colocados de forma que las ondas ultrasónica as atravesaran la pieza en el sentido transversal a las fibras.

Se procedió entonces a la lectura del tiempo (t) en microsegundos, necesario para que la onda ultrasónica atravesara la pieza de madera seca y haya sido recibida por el transductor de recepción, hasta que el equipo estabilice la lectura. Posteriormente, en posesión de esas lecturas y de la longitud del tramo
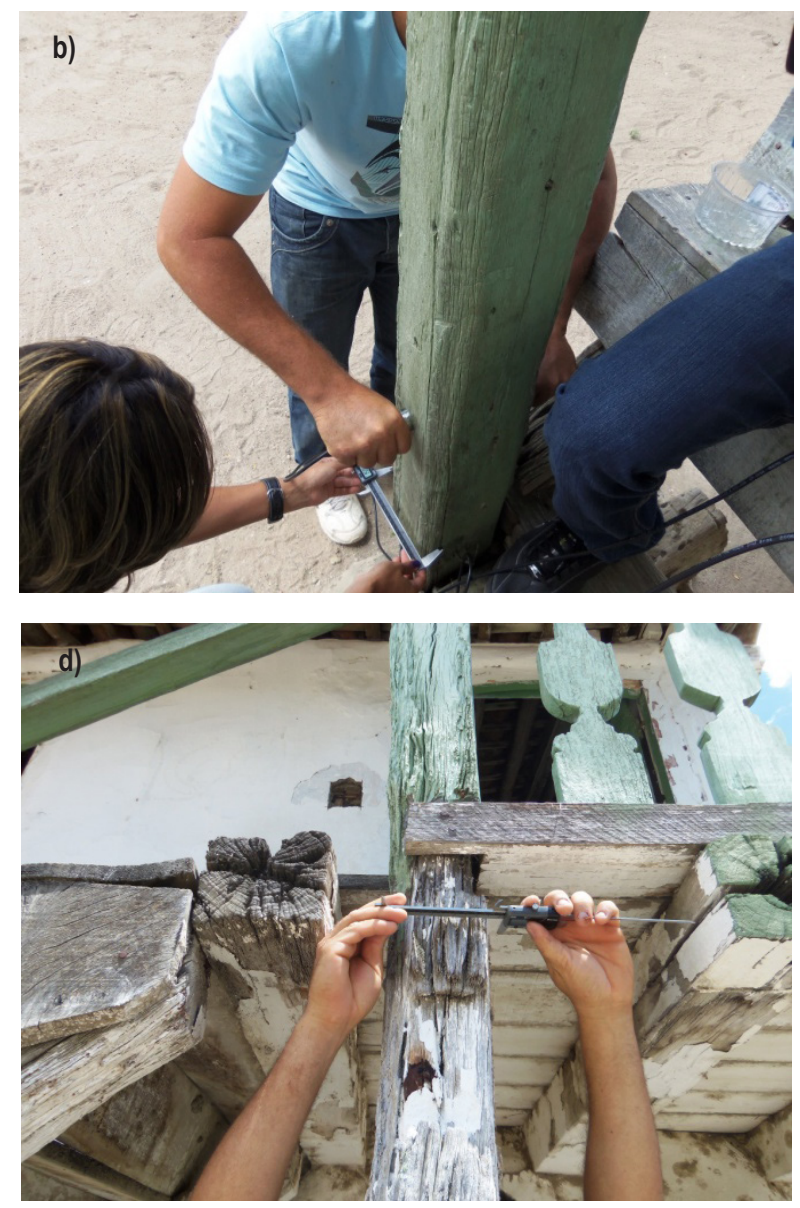

recorrido (L), se calculó la velocidad de propagación para cada pieza de madera, generando gráfico, que balizaron la identificación de los puntos más críticos de cada pieza.

Los ensayos se realizaron midiendo el tiempo de propagación de la propagación de la onda ultrasónica.

Para a determinación de las velocidades será utilizada la ecuación 1 :

$V=L / t$

Donde:

$V=$ velocidad de propagación de la onda $(\mathrm{m} / \mathrm{s})$

$\mathrm{L}=$ distancia conocida $(\mathrm{m})$

$\mathrm{T}$ = tiempo que tarda la onda ultrasónica de un transductor a otro (s).

Después del análisis de los datos de los pilares se elaboró la tabla 1, la cual establece tres intervalos para el diagnóstico de la madera y toma de decisión sobre el material. 
Tabla 1. Ranuras de variación de la velocidad de propagación de la onda ultrasónica en el sentido transversal y necesidad o no de intervención en la pieza de madera.

\begin{tabular}{lll}
\hline $\begin{array}{l}\text { Rango de variación de la velocidad en la dirección } \\
\text { transversal }(\mathrm{m} / \mathrm{s})\end{array}$ & Icono & Acción propuesta \\
\hline $0<1000$ & $\bullet$ & Sustitución \\
$1000<2000$ & & Reparación \\
$\geq 2000$ & $\bullet$ & Pieza sana \\
\hline
\end{tabular}

Estos intervalos fueron definidos de acuerdo con lo que preconiza la NBR 15521, y considerando además una compilación de los datos obtenidos por Nogueira (2003), que obtuvo una relación entre los valores medios de las velocidades de propagación de la onda de ultrasonido obtenidos en las tres direcciones consideradas (longitudinal, radial y tangencial) de 5 : 2: 1,5 respectivamente. Basados también en Beall (2002) que obtuvo una velocidad de propagación de las ondas ultrasónicas de $2000 \mathrm{~m} / \mathrm{s}$ en la dirección transversal para maderas exentas de defectos y en la condición seca.

\section{RESULTADOS Y DISCUSIÓN}

Los comportamientos de los gráficos de los pilares de uno al siete se verifican en la Figura 4. De acuerdo con esa figura en consonancia con la Tabla 2, se verificó que la diferencia entre la velocidad de propagación de la onda observada en la madera sana y la velocidad en las piezas con algún grado de compromiso puede ser un indicativo de mantenimiento, reparación o sustitución de la pieza.

Figura 4. Valores de la velocidad de propagación de la onda ultrasónica en los pilares uno al siete de la fachada de la Casa Grande de la Hacienda Cachoeira do Taépe

Pilar 1

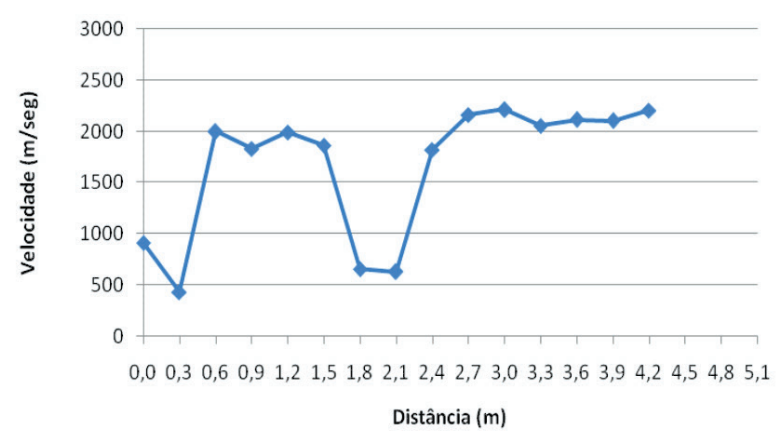

Pilar 3

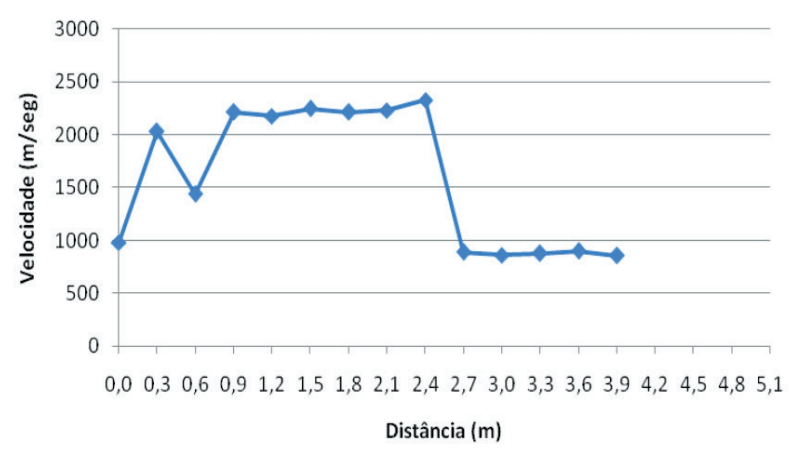

Pilar 2

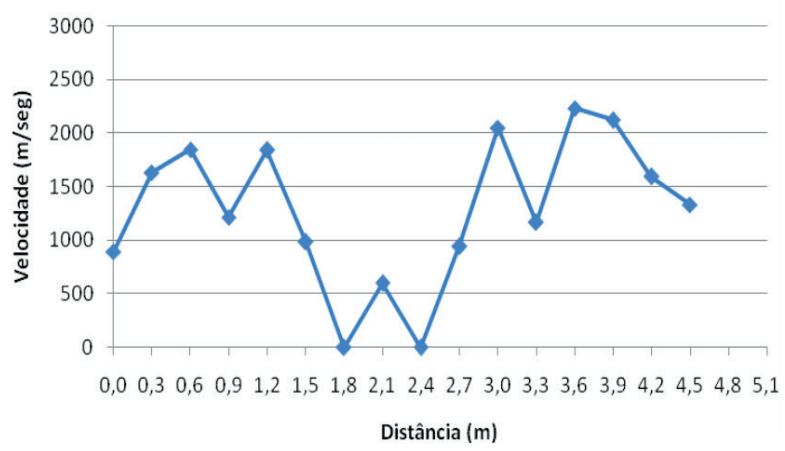

Pilar 4

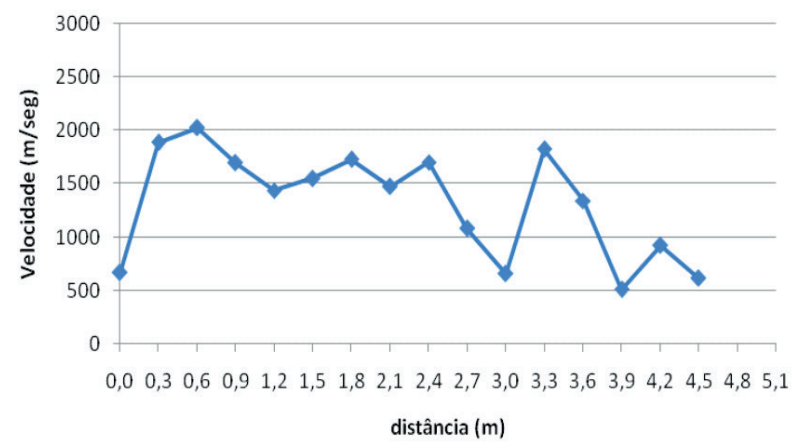


Pilar 5

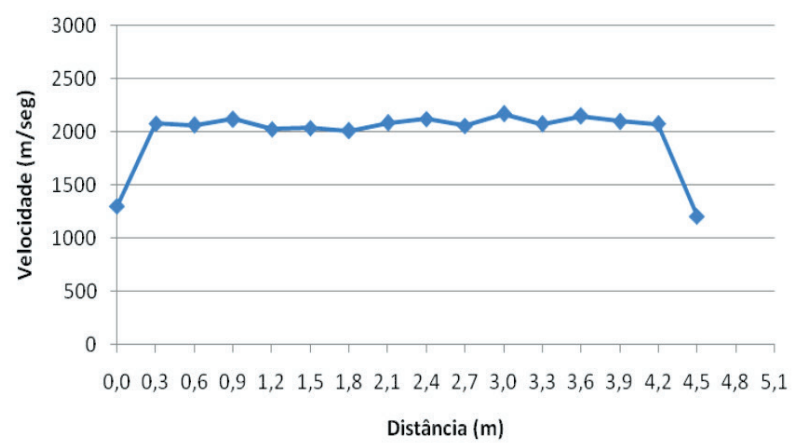

Pilar 6

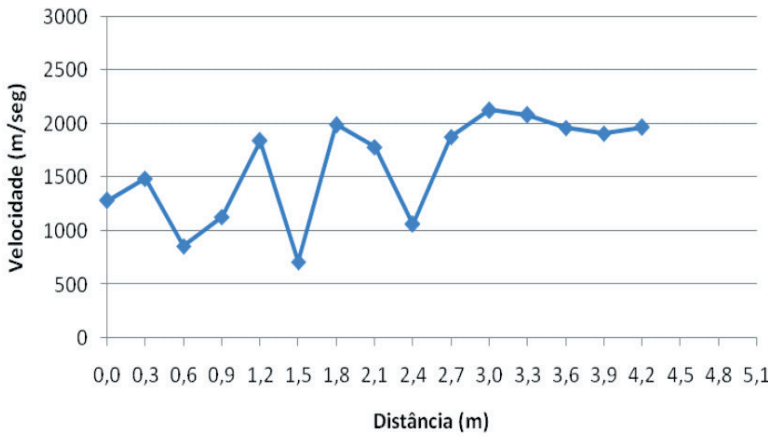

Pilar 7

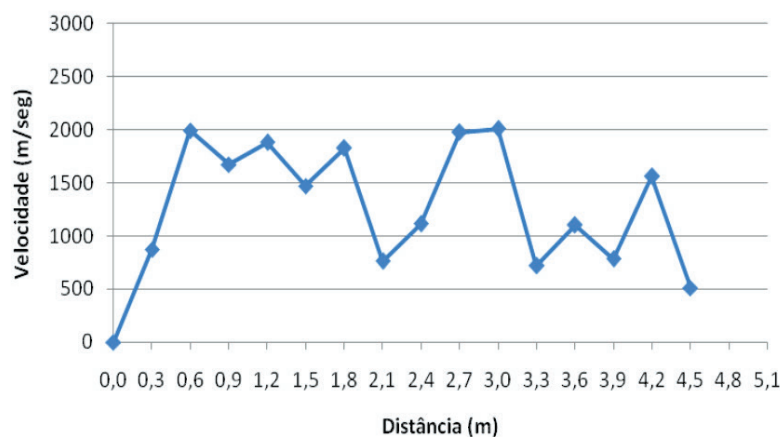

Tabla 2. Valores de la velocidad de propagación de la onda ultrasónica en los pilares de madera de la Casa Grande de la Hacienda Cachoeira do Taépe - Surubim - PE.

\begin{tabular}{|c|c|c|c|c|c|c|c|c|c|c|c|c|c|c|}
\hline \multirow{3}{*}{$\begin{array}{c}\text { Distância (m) } \\
0 \text { (base) }\end{array}$} & \multicolumn{14}{|c|}{ Velocidad de la Propagación de la onda ultrasonida (m/s) } \\
\hline & \multicolumn{2}{|c|}{$\mathrm{P} 7$} & \multicolumn{2}{|r|}{ P6 } & \multicolumn{2}{|r|}{ P5 } & \multicolumn{2}{|c|}{$\mathrm{P} 4$} & \multicolumn{2}{|r|}{$\mathrm{P} 3$} & \multicolumn{2}{|r|}{$\mathrm{P} 2$} & \multicolumn{2}{|r|}{$\mathrm{P} 1$} \\
\hline & O & & 0 & 1284 & O & 1303 & O & 668 & O & 982 & O & 893 & 0 & 912 \\
\hline 0,3 & O & 875 & 0 & 1490 & O & 2077 & O & 1888 & O & 2032 & O & 1635 & 0 & 434 \\
\hline 0,6 & 0 & 1994 & 0 & 860 & 0 & 2061 & 0 & 2023 & 0 & 1436 & 0 & 1849 & 0 & 1999 \\
\hline 0,9 & 0 & 1672 & 0 & 1133 & 0 & 2118 & 0 & 1696 & 0 & 2212 & 0 & 1215 & 0 & 1832 \\
\hline 1,2 & 0 & 1878 & 0 & 1843 & 0 & 2023 & 0 & 1438 & 0 & 2178 & 0 & 983 & 0 & 1992 \\
\hline 1,5 & 0 & 1472 & 0 & 713 & 0 & 2038 & 0 & 1551 & 0 & 2244 & 0 & 0 & 0 & 1861 \\
\hline 1,8 & 0 & 1826 & 0 & 1993 & 0 & 2010 & 0 & 1728 & 0 & 2214 & 0 & 600 & 0 & 658 \\
\hline 2,1 & 0 & 763 & 0 & 1784 & 0 & 2083 & 0 & 1471 & 0 & 2228 & 0 & 0 & 0 & 631 \\
\hline 2,4 & 0 & 1111 & 0 & 1063 & 0 & 2122 & 0 & 1701 & 0 & 2327 & 0 & 942 & 0 & 1821 \\
\hline 2,7 & 0 & 1974 & 0 & 1879 & 0 & 2056 & 0 & 1082 & 0 & 886 & 0 & 2050 & 0 & 2163 \\
\hline 3,0 & 0 & 2009 & 0 & 2129 & 0 & 2168 & 0 & 657 & 0 & 863 & 0 & 1166 & 0 & 2213 \\
\hline 3,3 & 0 & 717 & 0 & 2086 & 0 & 2075 & 0 & 1821 & 0 & 876 & 0 & 2235 & 0 & 2055 \\
\hline 3,6 & 0 & 1102 & 0 & 1963 & 0 & 2149 & 0 & 1342 & 0 & 898 & 0 & 2124 & 0 & 2116 \\
\hline 3,9 & 0 & 784 & 0 & 1913 & 0 & 2097 & 0 & 508 & 0 & 858 & 0 & 1595 & 0 & 2101 \\
\hline 4,2 & 0 & 1562 & 0 & 1966 & & & 0 & 923 & 0 & 816 & 0 & 1333 & 0 & 2206 \\
\hline 4,5 & 0 & 514 & 0 & 2083 & & & 0 & 616 & 0 & 1207 & & & & \\
\hline 4,8 & & & 0 & 1027 & & & & & & & & & & \\
\hline
\end{tabular}

Shaji et al. (2000), utilizando como referencia los valores para la velocidad de propagación obtenidos en piezas sanas, consideran que una pérdida mayor al
$30 \%$ en la velocidad de la onda en las piezas atacadas en relación a las sanas, exigirá el mantenimiento de ese material. Según Calegari et al. (2007) y Calegari et al. 
(2008) la masa específica y el contenido de humedad, así como el grado de deterioro influencian directamente en la propagación de las ondas de ultrasonido.

Stangerlim (2015) afirma que madera en contacto con el suelo tiene una mayor susceptibilidad al deterioro acarreando así menor velocidad de propagación de las ondas de ultrasonido. De una manera geral, se observó que las porciones más bajas de los pilares analizados presentaban el mayor nivel de degradación debido al contacto directo de la madera con el suelo. Por lo tanto, los datos observados en la base de los pilares son los más bajos.

El gráfico del pilar uno presenta un comportamiento que expresa claramente los puntos más críticos, ocurriendo una disminución de valores principalmente en la parte inferior y central del material. Demostrado en la distancia de 0.3, 1.8 y 2.1 metros valores de 434, 658 y $631 \mathrm{~m} / \mathrm{s}$, respectivamente, necesitando una mayor atención en esas porciones y posibles reparaciones en esos lugares, ya que en la parte superior del material se comprueban valores por encima de $2000 \mathrm{~m} / \mathrm{s}$.

Mientras que en el pilar cinco, se observaron valores superiores a $2000 \mathrm{~m} / \mathrm{s}$ casi en la totalidad de la pieza, indicando la sanidad del elemento estructural. Con apenas un punto crítico en los primeros 30 centímetros a partir de la base, debido a la exposición a agentes biológicos y físicos y presentando velocidad en torno a los $1300 \mathrm{~m} / \mathrm{s}$. En ese contexto, ese pilar debe permanecer, pero con el mantenimiento total de la pieza y realizando una intervención en el punto crítico.

El pilar tres tiene comportamiento con velocidad un poco inferior a $1000 \mathrm{~m} / \mathrm{s}$ en la porción superior de la sustentación y un descenso de la velocidad de propagación de la onda en la base, a pesar de presentar detalle constructivo por falta de mantenimiento preventivo, no escapó del ataque de los agentes físicos y biológicos. Gatto et al (2012) alega que cuando se compara la velocidad de propagación de las ondas ultrasónicas de las muestras de suelo sano con las atacadas por cualquiera de los agentes xilófagos se observó que la madera atacada presentó una velocidad de propagación menor.

El pilar seis indica un comportamiento similar en la porción central de la pieza con valores entre 713 y $860 \mathrm{~m} / \mathrm{s}$ y que muestra un rendimiento oscilante entre 1110 y $2100 \mathrm{~m} / \mathrm{s}$ a lo largo de la longitud de la pieza. Según Stangerlim et al. (2015), esta discontinuidad está relacionada a la presencia de zonas de pudrición 0 de cavidades formadas por insectos. Por lo tanto, estos pilares deben mantenerse en uso, precisando reparaciones puntuales y mantenimiento para su permanencia en la estructura.

De acuerdo con los datos, imágenes, valores de la velocidad de propagación de la onda y gráficos, se verifica un desempeño crítico en los pilares 2, 4 y 7 , debido a la reducción de la velocidad.

Se observa que hay una conducta grave en el pilar dos, principalmente en la porción intermedia de la pieza en las distancias de 1.2 a 2.4 metros y en la base del pilar con valores entre cero y $983 \mathrm{~m} / \mathrm{s}$. En las distancias de $1.5 \mathrm{~m}$ y $2.1 \mathrm{~m}$ no hubo lectura del equipo y el valor del tiempo de propagación en cero m/s está relacionado con la disipación de la onda e indica un posible punto donde la degradación del material es severa, una vez que el aire es Un pésimo conductor. Según Carrasco et al. (2012), la onda no se propaga en espacios vacíos y estos obstáculos pueden indicar ataque de insectos xilófagos. Por consiguiente, debe sustituirse dicho pilar.

El pilar cuatro expone los puntos de mayor fragilidad de la estructura, ya que los puntos manifiestan una variación de valores en el transcurso de las distancias. Evidenciando en la parte superior, la cual detiene valores entre 508 a $923 \mathrm{~m} / \mathrm{s}$, una reducción a su velocidad ocasionada por exposición a agentes patógenos y físicos. Necesitando reparaciones puntuales, pudiendo llevar la sustitución.

El pilar siete trae el mismo análisis de las demás piezas, presentando una gran variación a lo largo de la pieza, raramente llegando a $2000 \mathrm{~m} / \mathrm{s}$, con valores bajos en la velocidad de propagación de la onda en las extremidades superior e inferior del elemento, expuestas a acciones de las intemperies. Comprobando la urgencia en reparaciones y sustitución en los extremos de la pieza.

Los comportamientos de los pilares dos, cuatro y siete pueden ser corroborando por Reinprecht (2009), que al analizar las vigas de la Basílica de Egidius en Bardejov en Eslovaquia, determinó velocidades de propagación de ondas ultrasónicas en el rango comprendido entre 300 y $400 \mathrm{~m} / \mathrm{s}$ en las extremidades De las piezas analizadas, las cuales estaban gravemente atacadas por podredumbre parda y tenían visibles galerías de insectos. Ya en la parte central de las piezas, donde la incidencia de ataque de 
hongos e insectos era menos evidente, la velocidad de propagación de la onda quedó entre $700-1100 \mathrm{~m} / \mathrm{s}$.

Cuando el pilar o viga tenga la indicación de permanecer en la edificación, pero presentar partes deterioradas, deberán ser aplicadas prótesis. La pieza lesionada deberá ser cortada en punto sano, a aproximadamente $20 \mathrm{~cm}$ del tramo degradado y recibirá la enmienda, después de la inmunización de las partes de contacto.

Las condiciones ambientales actuaron en la construcción de manera continua, agresiva y inconstante, ya sea por el proceso de desgaste y / 0 pérdidas. El daño encontrado y lo arruinamiento puntual más significativo fueron causados por la actuación (0 por la ausencia) del propio hombre.

En vista de esto, el análisis del estado de conservación apunta las causas centradas en condiciones que actúan sobre los elementos constitutivos, de manera interdependiente: la presencia inadecuada de agua en las estructuras; infestación por agentes biológicos xilófagos y presencia humana. Además, con el desconocimiento de los atributos de los elementos de esta construcción y estas condiciones se ven agravados por la falta de mantenimiento.

Así está claro que los ensayos no destructivos con uso de ultrasonidos clasificaron las piezas estructurales de madera de manera satisfactoria para la localización de las regiones potencialmente atacadas en las estructuras de madera de construcciones históricas y sin ningún daño. Permitiendo un incremento en la calidad y la competitividad de estos materiales leñosos. Además, con este método es posible anticiparse a futuros problemas que podrían amenazar la estabilidad global de la estructura y impedir la sustitución innecesaria de elementos de la integridad de la edificación.

Las piezas estructurales evaluadas, en general, necesitan una intervención urgente en el sentido de promover el mantenimiento de la pieza, aunque sean en puntos específicos, pues presentan riesgos a los habitantes, correspondiendo a los órganos competentes ya que se trata de una edificación tumbada como Patrimonio histórico y consecuente restauración del patrón arquitectónico local, ya son atributos relacionados a la preservación de la imagen tanto el propio edificio, como el ejemplar de la arquitectura rural colonial en Pernambuco.

\section{REFERENCIAS}

Abreu, L. B.; Lima, J. T.; Rabelo, G. F.; Gomes, F. C.; Trugilho, P. F.; Eloy, F. S. Avaliação não destrutiva de estruturas de madeiras em edifício histórico de Tiradentes, MG. Revista Cerne, 2013, 19, 3, 481-487.

Associação Brasileira de Normas Técnicas. Ensaios não destrutivos - Ultra-som - Classificação mecânica de madeira serrada de dicotiledôneas (NBR 15521). Rio de Janeiro: $A B N T, 2007,7 \mathrm{p}$.

Associação Brasileira de Normas Técnicas. Projeto de estruturas de madeira (NBR 7190). Rio de Janeiro: ABNT, 1997, $107 \mathrm{p}$.

Ballarin, A. W.; Nogueira, M. Determinação do módulo de elasticidade da madeira juvenil e adulta de Pinus taeda por ultra-som. Engenharia Agrícola, 2005, 25, 1 , 19-28.

Beall, F. C. Overview of the use of ultrasonic technologies in research on wood properties. Wood Science and Technology, 2002, 36, 3, 197-212.

Calegari, L.; Stangerlin, D. M.; Santini, E. J.; Haselein, C. R.; Longhi, S. J.; Carmo, P. I. O.; Silva Filho, L. C. P.; Gatto, D. A. Avaliação de alguns fatores influentes na velocidade ultra-sônica na madeira. Revista Floresta, 2008, 38, 4, 607-615.

Calegari, L.; Stangerlin, D. M.; Santini, E. J.; Haselein, C. R.; Longhi, S. J.; Carmo, P. I. O.; Silva Filho, L. C. P.; Gatto, D. A. Monitoramento do teor de umidade de madeiras de Pinus elliottii Engelm. e Eucalyptus grandis W. Hill ex Maiden, sob diferentes temperaturas de secagem, através do ultra-som. Revista Ciência Florestal, 2007, 17, 4, 399-408.

Carrasco, E. V. M.; Teixeira, A. R.; Azevedo Jr, A. P.; Moreira, L. E. Metodología para análisis de la integridad de estructuras históricas de madera atacadas por insectos xilófagos por medio del ultrasonido. Revista Forestal Mesoamericana Kurú, 2012, 6, 16, 17-25.

Dantas, F. S. Patrimônio cultural, direito e meio ambiente: um debate sobre a globalização, cidadania e sustentabilidade [recurso eletrônico] / Juliano Bitencourt Campos, Daniel Ribeiro Preve, Ismael Francisco de 
Souza, organizadores - Curitiba: Multideia, 2015; v. I, 256p.

Gatto, D. A. Gonçalves, M. R. F.; Mattos, B. D.; Calegari, L.; Stangerlin, D. M. Estimativa da deterioração da madeira de assoalho de prédio histórico por meio de ondas ultrassônicas. Revista Cerne, 2012, 8, 4, 651 656.

Liñán, C. R; Conde, M. J. M.; Hita, R.; Gálvez, F. P. Inspección mediante técnicas no destructivas de un edificio histórico: oratorio San Felipe Neri (Cádiz). Informes de la Construcción, 2011, 63, 521, 13-22.

Martins, S. C. F. Arquitetura da terra roxa: inspeções em peças de madeira de patrimônio histórico rural utilizando ultrassom. 2011. (Tese de Doutorado) Universidade de São Paulo, São Paulo, Brasil. 169 p.

Muraro, C. L. Projeto de Restauração da Casa-Grande da Fazenda Cachoeira do Taepe: 0 edifício e unidade agropastoril. 2013. Dissertação (mestrado profissional em Conservação e Restauração de Monumentos e Núcleos Históricos) - Faculdade de Arquitetura e Urbanismo, Universidade Federal da Bahia, Salvador, Brasil. 447p.

Nogueira, M.; Ballarin, A. W. Efeito da idade na qualificação mecânica da madeira serrada de Eucalyptus sp. com uso de ensaios não-destrutivos. Revista Energia na Agriculltura, 2008, 23, 3, 74-94.
Oliveira, L. A. Metodologia para desenvolvimento de projeto de fachadas leves. 2009. (Tese de Doutorado) - Universidade de São Paulo, São Paulo, Brasil. 287 p.

Reinprecht, L. Diagnostic of the degraded zones for fir beam situated in the St. Egidiu's Basilica in Bardejov. Forestry and Wood Technology, 2009, 7, 201-207.

Secco, C. B.; Gonçalves, R.; Cerri, D. G. P.; Vasques, E. C.; Batista, F. A. F. Behavior of ultrasonic waves in wood with presence of holes. Cerne, 2012, 18, 3, 507514.

Shaji, T. Shaji, T.; Somayaji, S.; Mathews, M. S. Ultrasonic pulse velocity technique for inspecion and evaluation of timber. Journal of Materials in Civil Engineering, 2000, 12, 2, 180-185.

Stangerlin, D. M.; Gatto, D. A.; Melo, R. R.; Calegari, L.; Vivian, M. A.; Castelo, P. A. R. Beltrame, R. Uso do ultrassom para estimativa das propriedades mecânicas da madeira de Peltophorum dubium. Revista Ciência da Madeira (Brazilian Journal of Wood Science), 2010, 1, 2, 44-53.

Stangerlin, D. M.; Corassa, J. N.; Gatto, D. A.; Pereira, R. L.; Castelo, P. A. R. Caracterização mecânica de madeiras deterioradas em campo por meio de ultrassom e flexão estática. Comunicata Scientiae, 2015. 6, 3, 365-372.

Weiler, M.; Missio, A. L.; Gatto, D. A.; Güths, W. G. Nondestructive evaluation of wood decayed by xylophagous organisms. Materials Research, 2013, 16, 5, 1203-1213. 